\title{
Conceptual similarity promotes generalization of higher order fear learning
}

\author{
Joseph E. Dunsmoor, Allison J. White, and Kevin S. LaBar ${ }^{1}$ \\ Center for Cognitve Neuroscience and Department of Psychology and Neuroscience, Duke University, Durham, \\ North Carolina, 27708, USA
}

\begin{abstract}
We tested the hypothesis that conceptual similarity promotes generalization of conditioned fear. Using a sensory preconditioning procedure, three groups of subjects learned an association between two cues that were conceptually similar, unrelated, or mismatched. Next, one of the cues was paired with a shock. The other cue was then reintroduced to test for fear generalization, as measured by the skin conductance response. Results showed enhanced fear generalization that correlated with trait anxiety levels in the group that learned an association between conceptually similar stimuli. These findings suggest that conceptual representations of conditional stimuli influence human fear learning processes.
\end{abstract}

The idea that many fears originate from an association between neutral and aversive stimuli has continued to serve as the basis for influential accounts of human anxiety disorders (Bouton 2002; Mineka and Zinbarg 2006). However, the nexus of many fears defy a straightforward explanation, since following an aversive experience other stimuli that are indirectly related to the experience oftentimes become feared as well. In some cases, generalizing an acquired fear following an emotional learning episode is predicated on an association between stimuli formed before the emotional experience occurred. This form of stimulus generalization is known as sensory preconditioning (SPC) (Brogden 1939; Kimmel 1977; Gewirtz and Davis 2000), and is an important process whereby the representation of a known stimulus is modified following a salient experience with a related stimulus.

A typical SPC procedure occurs in three phases: first, subjects learn to associate two neutral cues-for example, a bell and a light. Next, one of the cues, say the light, is associated with a biologically significant unconditioned stimulus (US), which leads to the development of a conditioned response (CR). The bell is called the preconditioned stimulus (PS) and the light is called the conditioned stimulus (CS). Finally, the PS is presented alone. Despite the fact that it has never predicted the US directly, the PS will often elicit a CR, suggesting that an association was formed during preconditioning between the PS and CS. The majority of SPC studies have been conducted in nonhuman animals (Brogden 1939; Rizley and Rescorla 1972). SPC effects have also been reported in a small number of human fear-conditioning experiments (White and Davey 1989; Vansteenwegen et al. 2000), but human research on this topic is very limited relative to experiments using first-order Pavlovian conditioning procedures. Consequently, the features that promote fear generalization between previously associated stimuli are unknown.

A well-known factor important for classical conditioning (Rescorla and Furrow 1977) and stimulus generalization overall is similarity to the CS (Pavlov 1927; McLaren and Mackintosh 2002). Classic experiments of stimulus generalization in animals (reviewed in Honig and Urcuioli 1981) have revealed orderly generalization gradients of conditioned responses that track perceptual similarity to the CS along a sensory dimension (e.g., Guttman and Kalish 1956). Prior fear-conditioning research has

'Corresponding author.

E-mail klabar@duke.edu; fax (919) 681-0815.

Article is online at http://www.learnmem.org/cgi/doi/10.1101/lm.2016411. demonstrated perceptual-based stimulus generalization in humans as well (Lissek et al. 2008; Dunsmoor et al. 2009). Yet, humans frequently acquire information about a salient object that goes beyond its physical details. Thus, in many cases generalization is likely based on other regularities extracted from prior experience, such as the conceptual or semantic properties of a stimulus (Maltzman 1977). While the ability to generalize learning between physically dissimilar objects is not unique to humans (e.g., Honey and Hall 1989; Herrnstein 1990), humans are particularly adept at linking objects together on the basis of conceptual similarity, even in the absence of definable common features (Medin 1989). However, it is unknown whether the conceptual relationship between object representations influences generalization of higher order fear learning.

This study employed an SPC paradigm in a between-subjects design to examine whether conceptual similarity of preassociated cues influences fear generalization. Because of our interest in models of anxiety, fear-relevant stimuli were used. The PSs-a picture of a spider and a picture of a wasp-were held constant across the three groups. In the first phase, these PSs were associated with conceptually related CSs (spider web and wasp nest, respectively), unrelated CSs (waste drums or hospital corridor, counterbalanced), or conceptually mismatched CSs (wasp nest and spider web, respectively; Fig. 1). Next, one CS (CS+) was paired with an electrical shock US while the other CS (CS-) was explicitly unreinforced. Finally, the two PSs were presented alone to test for generalization of fear responses, measured here as an increase in SCRs from preconditioning to the generalization test. The effects of SPC were assessed by comparing increases in the SCR to the stimulus preassociated with the $\mathrm{CS}+$ (referred to as the $\mathrm{PS}+$ ) versus the stimulus preassociated with the CS- (referred to as the PS -). We hypothesized that SPC effects would be graded according to conceptual similarity, being strongest in the related group, intermediate in the unrelated group, and weakest in the mismatched group. We also hypothesized that conceptually driven SPC effects would be related to trait anxiety levels, in line with prior research demonstrating a relationship between anxiety and fear conditioning (Lissek et al. 2005).

Differential SCRs between the CS + and CS - provided the primary index of fear conditioning (Fig. 2). Analysis of the acquisition data revealed a significant effect of stimulus $\left(F_{(1,86)}=160.63\right.$, $P<0.001)$ and trial $\left(F_{(5,430)}=16.86, P<0.001\right)$, as well as a significant stimulus type $(\mathrm{CS}+, \mathrm{CS}-)$ by trial (1-6) interaction $\left(F_{(5,430)}=13.68, P<0.001\right)$, with a linear and quadratic trend 
Pre-association Learning Groups
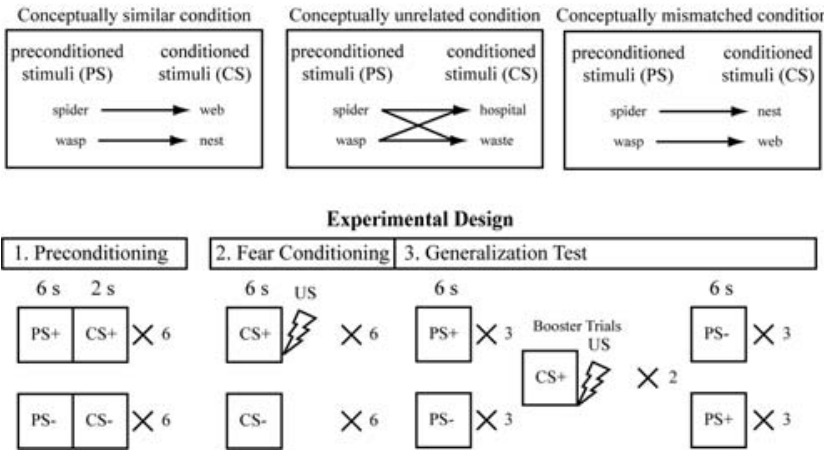

Figure 1. Schematic of experimental design. The preconditioned stimuli (PS) - pictures of a spider and wasp-were held constant across the three experimental groups. In the first phase of the experiment (sensory preconditioning), subjects learned the association between the PS and a conditioned stimulus (CS) that was either conceptually similar, unrelated, or conceptually mismatched. Subjects were instructed to predict which image would follow the offset of the PS as quickly and accurately as possible by pressing one of two buttons (counterbalanced between subjects). The task was intended to be simple to promote a strong association between the PS and its corresponding CS in all groups; consequently, all subjects correctly predicted the associated CS by the end of the preconditioning phase. Subjects were informed that no shocks would occur during this phase of the experiment, but were not informed of the PS-CS contingencies. In the next phase of the experiment (fear conditioning), one CS (CS + ) was reinforced by an aversive electrical shock unconditioned stimulus (US) on $100 \%$ of trials, while the other CS (CS - ) was never paired with the US. Subjects were informed at the start of fear conditioning that the task was to identify the image on the screen as quickly and accurately as possible by pressing one of two buttons (counterbalanced between subjects). Subjects were informed that the US would occur, but were not informed of the CS-US contingencies. For the conceptually similar and mismatched conditions, the CS+ and CS - stimuli were counterbalanced between subjects as either the spider web or the wasp nest. This ensured that the PS + and PS - were also counterbalanced between subjects as either the spider or the wasp. For the conceptually unrelated condition, the spider and wasp equally served as the PS+ (and PS-) in this condition as well. The final phase (generalization testing) immediately followed fear conditioning without a break, and subjects continued to identify the images on the screen by pressing one of two counterbalanced buttons as quickly and accurately as possible. After three PS + and three PS - trials, there were two CS+ trials paired with the shock. These "booster" trials were included to reduce habituation (e.g., Smith et al. 2006; Lim and Pessoa 2008), and data from these trials were not included in any analyses. Three additional PS + and PS - trials followed the "booster" trials.

$(P<0.01)$. There was no main effect of experimental group $\left(F_{(2,86)}=1.51, P=0.23\right)$, or any interactions involving group $(P s>0.05)$. As expected, post-hoc-dependent $t$-tests revealed that SCRs were overall greater to the CS + than the CS - as training progressed, being significant on trials $2-6$ (Ps $<0.01)$. Overall, fear acquisition was markedly similar among the three learning groups. The change in SCRs to the PS + and PS - from preconditioning to the generalization test served as the primary index of SPC. All trials from the preconditioning phase and the generalization test phase were included to calculate this difference score. ANOVA of the generalization test SCR data, using experimental group and stimulus type (PS+, PS -) as factors, revealed a main effect of group, $F_{(2,86)}=3.19, P<0.05$, and a main effect of stimulus, $F_{(1,86)}=11.37, P<0.001$, as well as an interaction between stimulus and group, $F_{(2,86)}=3.17, P<0.05$. Planned two-tailed $t$-tests comparing the PS + and PS - difference scores for each group revealed a significantly greater increase in SCRs to the PS + compared with the PS - in the conceptually similar condition, $t_{(29)}=2.85, P<0.01$, and the conceptually unrelated condition, $t_{(28)}=2.87, P<0.01$. Thus, the SPC manipulation was effective in these two groups. In addition, the magnitude of fear generalization was enhanced in the conceptually similar condition, as revealed by a significantly greater increase in SCRs to the PS + in this condition versus the conceptually unrelated condition, $t_{(28)}=2.38, P<0.05$. Responses to the PS - , however, were not significantly different, $P=0.11$. There was no difference in SCR scores to the PS + and PS - in the conceptually mismatched condition, $t_{(29)}=0.09, P>0.1$, suggesting that SPC was ineffective in this learning group.

During the generalization test, subjects were asked to identify the PS+ and PS - as quickly and accurately as possible.

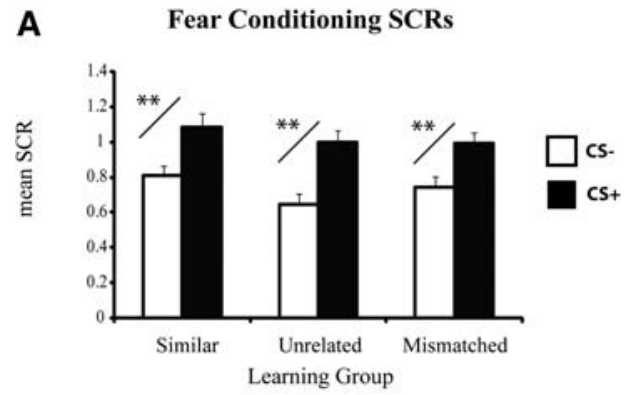

B Pre-to-Post fear conditioning SCRs

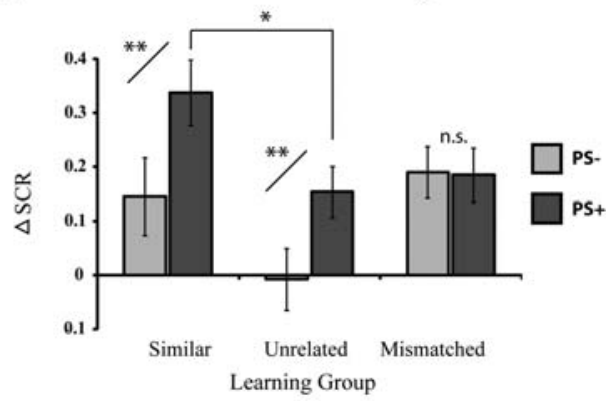

C

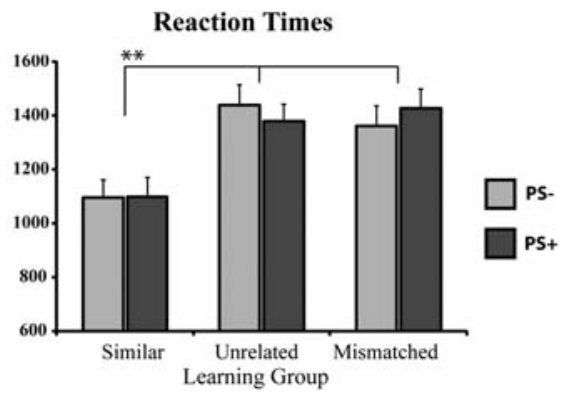

Figure 2. Behavioral results. (A) Differential mean skin conductance responses (SCR) during fear conditioning revealed acquisition of conditioned fear that was markedly similar across the three experimental groups. (B) Fear generalization was calculated as the change in SCRs from pre- to post-fear conditioning to the PS + and PS -. The change in SCRs was significantly greater to the PS + than the PS - in the conceptually similar and unrelated groups, but there was no difference in responses between the PS+ and PS - in the conceptually mismatched group. Responses to the PS+ were significantly enhanced in the conceptually similar versus conceptually unrelated condition, while responses to the PS - were not different across these groups. (C) Reaction times (RT) to identify the PS+ and PS - were significantly faster in the conceptually similar group versus the other two conditions. RT data from one subject from the conceptually unrelated condition was lost due to a technical issue. Error bars reflect standard error of the mean (SEM). ${ }^{*} P<0.05$, $* * P<0.01$ 
Analysis of the reaction time data demonstrated a main effect of group, $F_{(2,85)}=7.40, P<0.01$, but no effect of stimulus type and no interaction between stimulus and group. Post-hoc Bonferroni-corrected $t$-tests revealed that subjects in the conceptually similar condition exhibited faster RTs than subjects in the conceptually unrelated and conceptually mismatched conditions $(P s<0.005)$.

To explore the relationship between anxiety levels and higher order fear learning, trait anxiety levels were correlated with fear generalization to the PS + . In the conceptually similar condition, a significant correlation was found between fear generalization to the PS + and trait anxiety levels, $r_{(28)}=0.36, P<0.05$ (Fig. 3). In contrast, correlations with SCRs to the PS - were not significant, $r_{(28)}=0.25, P>0.05$, suggesting that anxiety levels were not associated with a general increase in arousal. We also examined whether the pre- to post-conditioning change in SCRs to the PS + were related to the level of fear conditioning (calculated as the differential SCR between the CS+ and CS-), and found that these measures were not significantly related, $r_{(28)}=$ $0.10, P>0.05$.

The results of the present experiment provide novel evidence that higher order fear learning in humans is influenced by the conceptual similarity of conditional cues. SPC effects were verified in both the conceptually similar and unrelated groups (PS $+>$ PS - responses), but the magnitude of responding to the $\mathrm{PS}+$ was greater in the conceptually similar group relative to the unrelated group. Moreover, RTs were faster during the fear generalization test in the conceptually similar group relative to the other groups, and only in this group was there a positive correlation between fear generalization to the PS + and trait anxiety. Subjects who learned the association between conceptually mismatched stimulus pairs showed only nonselective increases in SCRs during the generalization test. These findings were not attributable to group differences in initial fear learning or baseline responding. Altogether, the results support the hypothesis that conceptual relationships among stimuli modulate generalization of conditioned fear behaviors in humans.

The SCR findings are in line with the few previous investigations that have employed higher order fear learning procedures in humans (White and Davey 1989; Vansteenwegen et al. 2000; Hosoba et al. 2001; Gottfried and Dolan 2004). For instance, Vansteenwegen et al. (2000) observed SPC effects using pairs of affectively neutral faces and found that generalization was
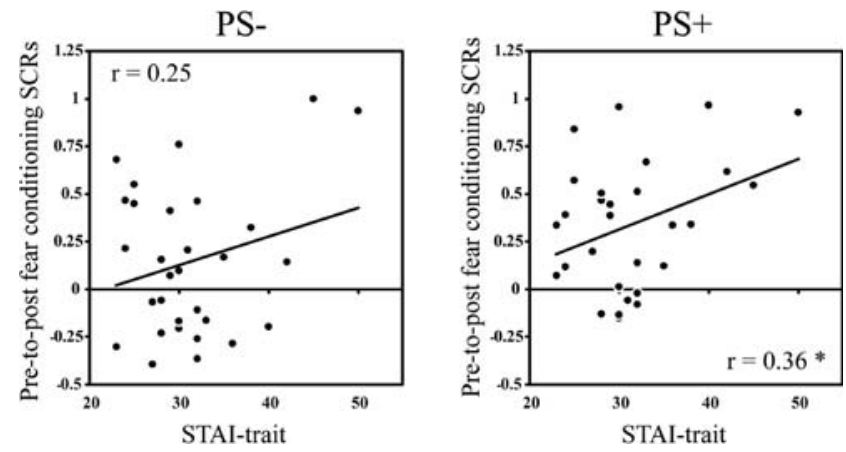

Figure 3. Correlations between fear generalization and trait anxiety scores for subjects in the conceptually similar condition. The change in SCRs to the PS + from pre- to post-fear conditioning were positively correlated with trait anxiety scores $(r=0.36, P<0.05)$, whereas trait anxiety was not significantly related to the change in SCRs to the PS - . There was no relationship observed between fear generalization and trait anxiety in the conceptually unrelated or conceptually mismatched groups. reduced when the PS+ was "preextinguished"-that is, when the PS was presented alone following preconditioning but prior to the fear conditioning phase. Therefore, a necessary condition for SPC may be an expectation that the PS portends delivery of the CS. In accord with this suggestion (Vansteenwegen et al. 2000), a PS and CS that are conceptually similar might serve to strengthen the interstimulus connection formed during preconditioning, which in turn facilitates fear generalization resulting from first-order fear learning. A similar process of stimulus belongingness has been posited to guide first-order fear conditioning in humans and nonhuman animals (Seligman 1970). For instance, in human fear conditioning fear-relevant CSs (e.g., snakes and spiders) appear to enter into an association with aversive electrical shocks more easily than fear-irrelevant CSs (e.g., flowers and mushrooms) (Öhman 2009). Importantly, SPC effects were observed in the conceptually unrelated condition as well, albeit at a lower magnitude. This finding suggests that conceptual similarity does not selectively induce generalization but rather enhances generalization to preassociated stimuli. Interestingly, SPC effects were not selective to the PS+ for the mismatched group, showing that conceptual relationships set boundary conditions for subsequent learning. The nonspecific form of fear generalization observed in the mismatched group may be attributed to two sources of generalization-sensory preconditioning and conceptual similarity-that operate in parallel on PS + and PS - representations. In this group, responses to the PS - are likely due to the conceptual relationship with the $\mathrm{CS}+$, while responses to the PS + are likely due to higher order fear learning processes resulting from SPC.

It is important to note that the relationship between objects in the conceptually similar condition was assumed to be conceptual in nature because these object pairs (e.g., spider and spider web) are characteristically associated (Medin 1989). However, it is also likely that these objects have frequently co-occurred in the learning history of our participants, and thus participants have been "preconditioned" prior to participation in this experiment. It is well known that experience shapes conceptual knowledge structures, so the effects observed likely include priors from both conceptual knowledge and direct learning history. If possible, future studies should attempt to dissect these influences, for instance by examining participants who have no direct prior experience with the stimuli. Because the CS+ and PS+ are assumed to be preassociated prior to the start of the experiment, it would also be interesting to determine whether a fear generalization paradigm that did not include an explicit preconditioning phase would yield comparable results, similar to studies that present perceptually varying stimuli following, but not prior to, acquisition training (e.g., Guttman and Kalish 1956).

The neural mechanisms supporting SPC effects have been explored predominately in rodents. These investigations have revealed that the hippocampus and surrounding regions are important for SPC, as lesions to the hippocampus (Talk et al. 2002), perirhinal cortex (Nicholson and Freeman 2000), CA1 (Port et al. 1987), and surrounding fibers (Port and Patterson 1984) block the effects of SPC while leaving classical conditioning intact (Port and Patterson 1984; Port et al. 1987). Associative learning models have implicated the hippocampal region as critical for several forms of conditioned learning that involve the representation of conditional stimuli (e.g., Gluck and Myers 1993; Schmajuk et al. 1996). SPC effects that include the transfer of fear value likely involve the amygdala as well (Gewirtz and Davis 2000), though the precise role of the amygdala in cognitive processes that involve higher order stimulus representations remains to be elucidated (Killcross et al. 1997; Mesulam 1998). In terms of the present findings, fear generalization between related object concepts may require integration between the 
amygdala, hippocampus, and posterior cortical areas important for coding the perceptual and conceptual properties of objects (Martin 2007). Future studies will be needed to resolve the role of corticolimbic structures in human fear generalization.

The overall lack of human behavioral research on SPC is somewhat surprising, given that influential conditioning-based models of anxiety have incorporated higher order fear learning processes to explain the etiology of several anxiety disorders (Rachman 1991; Davey 1992; Bouton et al. 2001; Mineka and Zinbarg 2006). Here, we observed an effect of trait anxiety on fear generalization within the conceptually similar condition, such that subjects with high anxiety expressed a greater increase in SCRs to the PS+ following fear conditioning. The ability to acquire and express conditional fears has long served as a model for clinical anxiety disorders (Watson and Rayner 1920; Eysenck 1976), and prior studies have indicated that clinically anxious subjects express broad generalization of conditioned fear responses (Lissek et al. 2010) and fail to limit fear behaviors to conditioned stimuli (reviewed in Lissek et al. 2005). Trait anxiety could mediate higher forms of fear learning through several processes, such as expectancy biases (Chan and Lovibond 1996) and heightened threat appraisal (Macleod and Cohen 1993), which could lead to the detection of generalized danger signals that are only indirectly related to the feared US. Future studies should examine whether clinical anxiety disorders are associated with a broadening of SPC effects, even in cases where the preassociated stimuli are less conceptually related.

Subjects were 113 healthy young adults. Data from 24 subjects was not included in the final analysis due to a lack of measurable SCR $(n=8)$ or a lack of differential fear conditioning SCRs between the CS + and CS $-(n=15)$. Removing these participants from analysis is justified because the study goal was to determine how fears generalized in individuals who acquired a differential fear response (i.e., evidence of fear learning was an inclusionary criterion). Data from one subject was excluded due to normalized fear generalization SCR scores that were 3 standard deviations from the group mean. The remaining 89 subjects (30 female; mean age \pm SEM: $19.05 \pm 0.1$; age range $18-23$ ) were randomly assigned to the conceptually similar, conceptually unrelated, or conceptually mismatched conditions. Subjects completed the Spielberger State-Trait Anxiety Inventory (STAI; Spielberger 1983) and a questionnaire assessing attitudes toward spiders (Klorman et al. 1974) and wasps. The wasp questionnaire was a slightly modified version of the validated spider questionnaire. All subjects fell below the exclusionary criterion of phobia scores, set at within 1 SD from the mean of patients with a specific phobia for spiders (Fredrikson 1983).

All images were pilot rated for valence and arousal, and matched for luminance and contrast. The hospital and waste barrel pictures were obtained from the International Affective Picture System (IAPS: \#7520, \#7079) (Lang et al. 2008). The experimental design is depicted in Figure 1. Details concerning the electrical stimulation and SCR analysis are described elsewhere (Dunsmoor et al. 2009). In brief, the electrical shock US was delivered to the right wrist and managed with the BIOPAC STM200 module (BIOPAC Systems) and set to a level deemed by each subject as "highly annoying, but not painful." SCRs were recorded from the middle phalanx of the second and third digits of the left hand using $\mathrm{Ag} / \mathrm{AgCl}$ electrodes connected to the BIOPAC MP150 system (BIOPAC System, Inc.) at $200 \mathrm{~Hz}$ and scored with AcqKnowledge software (BIOPAC Systems, Inc.). SCRs were considered related to the stimulus presentation if the trough-to-peak response (1) occurred at least $1 \mathrm{sec}$ after stimulus onset, (2) lasted between $0.5-5.0 \mathrm{sec}$, and (3) was $>0.02$ microsiemens $(\mu \mathrm{S})$. A response that did not meet these criteria was scored as zero. SCRs were square-root transformed for normalization. For analysis of fear generalization, mean SCRs to the PS+ and PS - during the preconditioning phase were subtracted from mean SCRs to the PS + and PS - during the generalization test for each subject. Statistical analysis included $3 \times 2$ repeated-measures ANOVA with experimental group (conceptually similar, conceptually unrelated, conceptually mismatched) as a between-subjects factor and stimulus type (CS + and CS - , or PS + and PS - ) as a withinsubjects factor. Planned two-tailed $t$-tests were conducted to examine a priori hypotheses and to determine differences between the stimulus types where appropriate. Results from statistical analyses were considered significant at an $\alpha$ level of 0.05 .

\section{Acknowledgments}

We thank Matthew Fecteau for assistance with the psychophysiological equipment. This project was supported by NSF grant 0745919 and NIH grant 2 P01 NS041328.

\section{References}

Bouton ME. 2002. Context, ambiguity, and unlearning: Sources of relapse after behavioral extinction. Biol Psychiatry 52: 976-986.

Bouton ME, Mineka S, Barlow DH. 2001. A modern learning theory perspective on the etiology of panic disorder. Psychol Rev 108: $4-32$.

Brogden WJ. 1939. Sensory pre-conditioning. J Exp Psychol 25: 323-332.

Chan CKY, Lovibond PF. 1996. Expectancy bias in trait anxiety. J Abnorm Psychol 105: 637-647.

Davey GCL. 1992. Classical-conditioning and the acquisition of human fears and phobias-a review and synthesis of the literatures. Adv Behav Res Ther 14: 29-66.

Dunsmoor JE, Mitroff SR, LaBar KS. 2009. Generalization of conditioned fear along a dimension of increasing fear intensity. Learn Mem 16: 460-469.

Eysenck HJ. 1976. The learning theory model of neurosis-a new approach. Behav Res Ther 14: 251-267.

Fredrikson M. 1983. Reliability and validity of some specific fear questionnaires. Scand J Psychol 24: 331-334.

Gewirtz JC, Davis M. 2000. Using Pavlovian higher-order conditioning paradigms to investigate the neural substrates of emotional learning and memory. Learn Mem 7: 257-266.

Gluck MA, Myers CE. 1993. Hippocampal mediation of stimulus representations-a computational theory. Hippocampus 3: 491-516.

Gottfried JA, Dolan RJ. 2004. Human orbitofrontal cortex mediates extinction learning while accessing conditioned representations of value. Nat Neurosci 7: 1145-1153.

Guttman N, Kalish HI. 1956. Discriminability and stimulus-generalization. J Exp Psychol 51: 79-88.

Herrnstein RJ. 1990. Levels of stimulus-control-a functional approach. Cognition 37: 133-166.

Honey RC, Hall G. 1989. Acquired equivalence and distinctiveness of cues. J Exp Psychol-Anim Behav Process 15: 338-346.

Honig WK, Urcuioli PJ. 1981. The legacy of Guttman and Kalish (1956)—25 years of research on stimulus-generalization. J Exp Anal Behav 36: $405-445$.

Hosoba T, Iwanaga M, Seiwa H. 2001. The effect of UCS inflation and deflation procedures on "fear" conditioning. Behav Res Ther 39: $465-475$.

Killcross S, Robbins TW, Everitt BJ. 1997. Different types of fear-conditioned behaviour mediated by separate nuclei within amygdala. Nature 388: 377-380.

Kimmel HD. 1977. Notes from Pavlovs Wednesdays-sensory preconditioning. Am J Psychol 90: 319-321.

Klorman R, Weerts TC, Hastings JE, Melamed BG, Lang PJ. 1974. Psychometric description of some specifici-fear questionnaires. Behav Ther 5: 401-409.

Lang PJ, Bradley MM, Cuthbert BN. 2008. International affective picture system (IAPS): Affective ratings of pictures and instruction manual. Technical Report A-8. University of Florida, Gainesville, FL.

Lim SL, Pessoa L. 2008. Affective learning increases sensitivity to graded emotional faces. Emotion 8: 96-103.

Lissek S, Powers AS, McClure EB, Phelps EA, Woldehawariat G, Grillon C, Pine DS. 2005. Classical fear conditioning in the anxiety disorders: A meta-analysis. Behav Res Ther 43: 1391-1424.

Lissek S, Biggs AL, Rabin SJ, Cornwell BR, Alvarez RP, Pine DS, Grillon C. 2008. Generalization of conditioned fear-potentiated startle in humans: Experimental validation and clinical relevance. Behav Res Ther 46: 678-687. 
Lissek S, Rabin S, Heller RE, Lukenbaugh D, Geraci M, Pine DS, Grillon C. 2010. Overgeneralization of conditioned fear as a pathogenic marker of panic disorder. Am J Psychiatry 167: 47-55.

Macleod C, Cohen IL. 1993. Anxiety and the interpretation of ambiguitya text comprehension study. J Abnorm Psychol 102: 238-247.

Maltzman I. 1977. Orienting in classical conditioning and generalization of the galvanic skin-response to words: An overview. J Exp Psychol Gen 106: $111-119$

Martin A. 2007. The representation of object concepts in the brain. Annu Rev Psychol 58: $25-45$

McLaren IPL, Mackintosh NJ. 2002. Associative learning and elemental representation: II. Generalization and discrimination. Anim Learn Behav 30: 177-200.

Medin DL. 1989. Concepts and conceptual structure. Am Psychol 44: 1469-1481.

Mesulam MM. 1998. From sensation to cognition. Brain 121: 1013-1052.

Mineka S, Zinbarg R. 2006. A contemporary learning theory perspective on the etiology of anxiety disorders-It's not what you thought it was. Am Psychol 61: 10-26.

Nicholson DA, Freeman JH. 2000. Lesions of the perirhinal cortex impair sensory preconditioning in rats. Behav Brain Res 112: 69-75.

Öhman A. 2009. Of snakes and faces: An evolutionary perspective on the psychology of fear. Scand J Psychol 50: 543-552.

Pavlov IP. 1927. Conditioned Reflexes. Oxford University Press, London, UK.

Port RL, Patterson MM. 1984. Fimbrial lesions and sensory preconditioning. Behav Neurosci 98: 584-589.

Port RL, Beggs AL, Patterson MM. 1987. Hippocampal substrate of sensory associations. Physiol Behav 39: 643-647.
Rachman S. 1991. Neo-conditioning and the classical theory of fear acquisition. Clin Psychol Rev 11: 155-173.

Rescorla RA, Furrow DR. 1977. Stimulus similarity as a determinant of Pavlovian conditioning. J Exp Psychol-Anim Behav Process 3: 203-215.

Rizley RC, Rescorla RA. 1972. Associations in second-order conditioning and sensory preconditioning. J Comp Physiol Psychol 81: 1-11.

Schmajuk NA, Lam YW, Gray JA. 1996. Latent inhibition: A neural network approach. J Exp Psychol-Anim Behav Process 22: 321-349.

Seligman ME. 1970. On the generality of the laws of learning. Psychol Rev 77: $406-418$

Smith SD, Most SB, Newsome LA, Zald DH. 2006. An emotion-induced attentional blink elicited by aversively conditioned stimuli. Emotion 6: $523-527$.

Spielberger CD. 1983. Manual for the State-Trait Anxiety Inventory. Consulting Psychologists Press, Palo Alto, CA.

Talk AC, Gandhi CC, Matzel LD. 2002. Hippocampal function during behaviorally silent associative learning: Dissociation of memory storage and expression. Hippocampus 12: 648-656.

Vansteenwegen D, Crombez G, Baeyens F, Hermans D, Eelen P. 2000. Pre-extinction of sensory preconditioned electrodermal activity. QJExp Psychol B 53: 359-371.

Watson JB, Rayner R. 1920. Conditioned emotional reactions. J Exp Psychol 3: $1-14$.

White K, Davey GCL. 1989. Sensory preconditioning and UCS inflation in human fear conditioning. Behav Res Ther 27: 161-166.

Received September 17, 2010; accepted in revised form December 8, 2010. 


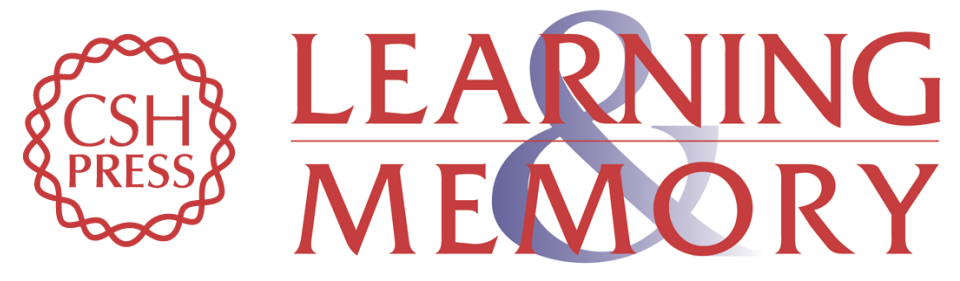

\section{Conceptual similarity promotes generalization of higher order fear learning}

Joseph E. Dunsmoor, Allison J. White and Kevin S. LaBar

Learn. Mem. 2011, 18:

Access the most recent version at doi:10.1101//m.2016411

References This article cites 44 articles, 2 of which can be accessed free at:

http://learnmem.cshlp.org/content/18/3/156.full.html\#ref-list-1

License

Email Alerting Receive free email alerts when new articles cite this article - sign up in the box at the Service top right corner of the article or click here. 